\title{
Fatores Genéticos e Ambientais que Influenciam Algumas Características de Reprodução e Produção de Leite em Cabras no Distrito Federal ${ }^{1}$
}

\author{
Guilherme Soares Filho², Concepta McManus ${ }^{3}$, Arthur da Silva Mariante ${ }^{4}$
}

\begin{abstract}
RESUMO - Este trabalho foi desenvolvido no Distrito Federal em dois capris, utilizando-se dados de 772 parições, com o objetivo de avaliar os fatores ambientais e genéticos que influenciam a reprodução e a produção leiteira em caprinos das raças Saanen, Parda Alpina, Toggenburg e seus mestiços, no período de 1995 a 1997. Os dados foram analisados usando o programa SAS com os procedimentos GLM e as herdabilidades foram estimadas usando o modelo animal individual pelo programa MTDFREML. As características avaliadas foram idade ao primeiro parto (IPP), intervalo de partos (IP), período de gestação (PG), produção de leite total (PLT) e duração da lactação (DL). O modelo estatístico incluiu como fatores fixos o capril, a raça, o mês e o ano de parto, o tipo de parto, o sexo da cria (macho e fêmea) e a covariável peso da cabra à cobertura. A raça Saanen teve a menor IPP e a maior PLT, enquanto a raça Toggenburg teve maior IP. As herdabilidades estimadas foram, em geral, baixas e variaram de acordo com a raça. Os fatores capril, raça, mês do parto e sexo da cria influenciaram os IP. A raça e o tipo de parto influenciaram a IPP. Somente a raça influenciou a PLT. Os resultados deste trabalho evidenciaram que existe grandeoportunidade para a melhoria no desempenho de caprinos no Distrito Federal, necessitando de controle mais rigoroso do rebanho e manejo para reduzir as IPP e os IP e aumentar os níveis de produção.
\end{abstract}

Palavras-chave: caprinos, duração da lactação, gestação, idade ao primeiro parto, intervalo de partos, produção de leite

\section{Genetic and Environmental Factors which Influence Reproduction and Milk Production Traits in Goats in the Federal District, Brazil}

\begin{abstract}
This study was carried out in the Federal District of Brazil on two goat farms, using data collected on 772 parturitions. The objective was to evaluate genetic and environmental factors, which influenced the reproduction and milk production of Saanen, Parda Alpine, Toggenburg goats and their crosses, between 1995 and 1997. The data were analysed using SAS (Statistical Analysis System, GLM procedures) and the heritabilities were estimated using MTDFREML with an Individual Animal Model. The studied traits were: age at first kidding (IPP), kidding interval (IP), gestation period (PG), total milk production (PLT) and lactation length (DL). The statistical model included fixed effects [farm, breed, month and year of kidding, type of parturition, sex of the kid (male, female)] and the covariate, dam weight at matting time. The Saanen breed had the lowest IPP and the highest PLT, while the Toggenburg breed had the highest IP. The heritabilities were low and dependent on the breed. The factors as farm, breed, month of kidding and sex of the kid affected the IP. The breed and the type of parturition affected the IPP. The breed only influenced the PLT. The results of this work evidenced that there is a high opportunity to improve the goat performances in Federal District, needing more rigor in herd control and management to reduce the IPP and IP and to increase the production levels.
\end{abstract}

Key Words: age at first kidding, gestation period, goats,kidding interval and milk production, lactation length

\section{Introdução}

A eficiência produtiva da pecuária leiteira é, em parte, determinada pela eficiência reprodutiva. Entre os parâmetros que permitem avaliar a eficiência reprodutiva e produtiva de um rebanho, destacam-se a idade ao primeiro parto, o intervalo de partos, o período de gestação, a produção de leite e a duração da lactação.

Os caprinos originários de países de clima temperado apresentam marcante influência quanto às vari- ações do fotoperíodo, já que iniciam seu ciclo reprodutivo anual em função da diminuição da intensidade de luz diária, o que se reflete significativamente na eficiência reprodutiva e produtiva (CHEMINEAU et al., 1993 e GALINA et al., 1995). O fotoperíodo e a temperatura são fatores principais de interferência na reprodução desses animais, sendo que o primeiro tem como interdependência a latitude, um caráter diretamente proporcional (CHEMINEAU et al., 1993). Na região Centro-Sul do Brasil, os animais apresentam-se poliéstricos estacionais, passando a ter estros

\footnotetext{
1 Parte da dissertação de Mestrado em Agronomia da Universidade de Brasília do primeiro autor, com bolsa da CAPES.

2 Professor da Faculdade da Terra de Brasília e aluno de Doutorado da Faculdade de Saúde da UnB. E.mail: guilherme@ftb.br

3 Professor da Faculdade de Agronomia e Medicina Veterinária, Universidade de Brasília. E.mail: concepta@unb.br

4 Pesquisador, EMBRAPA Recursos Genéticos e Biotecnologia, Brasília, DF. E.mail: mariante@cenargen.embrapa.br
} 
regulares apenas no verão e outono, o que os diferencia dos animais da região Nordeste, onde existe pouca variação no ciclo anual do fotoperíodo, devido à latitude, predominando os poliéstricos contínuos, (SIMPLÍ́CIO et al., 1986).

Observa-se que alto investimento e custo de manutenção são despendidos com as fêmeas desde o nascimento até o primeiro parto. No caso de cabritas de raças européias, estacionais quanto ao ciclo estral, este custo de manutenção é ainda mais elevado, uma vez que, dependendo da época de nascimento e da evolução do crescimento, os animais não concebem na primeira estação de monta, e seu primeiro parto só ocorre no segundo ano de vida (GONÇALVES et al., 1996). Conseqüentemente, animais com idades muito avançadas ao primeiro parto apresentam significativa redução em sua vida útil produtiva.

O intervalo de partos (IP) é definido como o período compreendido entre duas parições, sendo considerado um dos melhores parâmetros para medir a eficiência reprodutiva de um rebanho (TERRIL e FOOTE, 1987).

No Brasil, estudos com a espécie caprina demonstram que o intervalo de partos é alto em raças especializadas, devido ao efeito da estacionalidade reprodutiva nesses animais, enquanto para os caprinos nativos de regiões tropicais, onde a influência da sazonalidade é baixa, observam-se intervalos com melhores índices (GONÇALVES et al., 1996). O intervalo de partos é uma característica que pode interferir diretamente na rentabilidade de uma exploração. Além disso, limita a intensidade de seleção, uma vez que o prolongamento do intervalo de partos diminui o número de cabritos desmamados e aumenta o intervalo de gerações.

O período de gestação é definido como o tempo compreendido entre a concepção e o parto. A duração da gestação é geneticamente estabelecida para cada espécie, mas pode ser influenciada por diversos fatores e por suas interações (SIMPLÍCIO et al., 1990). Os fatores que influenciam o período de gestação são classificados em: 1) genéticos, como raça, genótipo da mãe e do feto (ILOEJE e VAN VLECK, 1978) e 2) ambientais, como estação do ano e alimentação (RUVUNA et al., 1987), sendo que ambos têm atividade fisiológica, geralmente com participação do sistema endócrino.

KENNEDY et al. (1982) relatam que diversos fatores podem influenciar a produção de leite, como, por exemplo, raça, alimentação, idade da matriz, número de partos, número de crias, número de ordenhas diárias e condições climáticas.

Na Califórnia, ALDERSON e POLLAK (1980) examinaram algumas características de produção em animais das raças Parda Alpina, La Mancha, Nubiana, Saanen e Toggenburg e encontraram fatores como idade, estação do ano e raça influenciando significativamente $(\mathrm{P}<0,001)$ a produção de leite. Provavelmente, estes resultados foram afetados pela distribuição de alimentos ao longo da lactação.

WOOD (1980), em seus estudos, descreveu que a duração da lactação é o período no qual o pico de produção de leite é mantido dentro da curva de lactação.

A duração da lactação é uma característica de interesse econômico estreitamente relacionado à produção de leite, além disso, define a forma da curva de lactação (SINGH et al., 1970). Por outro lado, é importante ressaltar que a persistência prolongada resultará em diminuição na eficiência reprodutiva do animal ao longo de sua vida produtiva.

O objetivo deste trabalho foi avaliar alguns fatores ambientais e genéticos que afetam a produção de leite e a reprodução de cabras em dois capris do Distrito Federal.

\section{Material e Métodos}

Os dados utilizados neste trabalho foram provenientes de dois capris no Distrito Federal, obtidos no período de 1995 a 1997. As observações consideradas neste estudo foram 504 registros de idade ao primeiro parto, 103 de intervalo de partos, $365 \mathrm{de}$ período de gestação, 298 de produção total de leite e 295 de duração da lactação, sendo 258 animais da raça Saanen, 270 da raça Parda Alpina, 145 da raça Toggenburg, 85 mestiços e 14 reprodutores distribuídos nos dois criatórios. O capril 1 possuía as raças Saanen, Parda Alpina, Toggenburg e mestiços, enquanto o capril 2, somente as raças Parda Alpina e mestiços.

A alimentação foi fornecida de acordo com as necessidades nutricionais dos animais nas diversas categorias. As fêmeas foram divididas em lotes por idade e receberam como volumoso capim-elefante (Pennisetum purpureum, Schum) ou silagem de milho (Zea mays L.), dependendo da estação do ano. Além disso, receberam em torno de $500 \mathrm{~g} / \mathrm{dia}$ de concentrado com mínimo de $18 \%$ de proteína bruta. Quando atingiam pesos que variavam de $32 \mathrm{a} 35 \mathrm{~kg}$, as fêmeas iniciaram a vida reprodutiva. Até o terço 
final de gestação, as cabras permaneceram recebendo a mesma alimentação, sendo que a partir daí passaram a receber concentrado com mínimo de $22 \%$ de proteína bruta numa quantidade de 600 a $800 \mathrm{~g} / \mathrm{dia}$. Os machos reprodutores receberam o mesmo volumoso das cabras e cerca de 400 a $600 \mathrm{~g} / \mathrm{dia}$ de concentrado com um mínimo de $20 \%$ de proteína bruta. Todas as categorias receberam uma mistura mineral e água à vontade.

Como as raças estudadas são poliéstricas estacionais, com o pico da estação reprodutiva nos meses de verão e outono, as duas fazendas utilizaram o sistema de reprodução programada composto pela indução do cio com luz artificial nos meses em que as fêmeas se apresentavam com anestro funcional. Como resultado, havia distribuição de partos durante todo o ano. O método de cobertura adotado foi o de monta natural controlada e inseminação artificial, em que todas as fêmeas foram submetidas à pesagem na cobertura.

Os dados foram constituídos de peso de cobertura da cabra (kg), mês (janeiro a dezembro) e ano (1995 a 1997) de cobertura, identificação do reprodutor (número ou nome), identificação do sexo da cria (macho ou fêmea) e tipo de parto (simples ou múltiplo), além de idade ao primeiro parto e partos subseqüentes, intervalo de partos, período de gestação, produção total de leite e duração da lactação.

As análises de variância foram realizadas pelo método dos quadrados mínimos, por intermédio de um modelo estatístico linear de classificação múltipla, com auxílio computacional do programa estatístico Statistical Analysis System/SAS usando o procedimento GLM (General Linear Model) (SAS,1985) e MTDFREML (Multiple Trait Derivate Free Restricted Maximun Likelihood), desenvolvido por BOLDMAN et al. (1993).

O modelo estatístico geral utilizado no procedimento GLM foi o seguinte:

$$
\begin{gathered}
\mathrm{Y}_{\mathrm{ijklmn}}=\mu+\mathrm{C}_{\mathrm{i}}+\mathrm{R}_{\mathrm{j}}+\mathrm{M}_{\mathrm{k}}+\mathrm{A}_{1}+\mathrm{T}_{\mathrm{m}}+\mathrm{S}_{\mathrm{n}}+b_{1} \\
\left(\mathrm{P}_{\mathrm{ijklmn}}-\overline{\mathrm{P}}\right)+b_{2}\left(\mathrm{P}_{\mathrm{ijklmn}}-\overline{\mathrm{P}}\right)^{2}+\mathrm{e}_{\mathrm{ijklmn}}
\end{gathered}
$$

em que $Y_{i j k l m n}$ é característica; $\mu$, média geral da característica; $\mathrm{C}_{\mathrm{i}}$, efeito fixo do $\mathrm{i}_{\text {ésimo }}$ capril; $\mathrm{i}=1,2$; $\mathrm{R}_{\mathrm{j}}$, efeito fixo da $\mathrm{j}_{\text {ésima }}$ raça; $\mathrm{j}$, Saanen, Parda Alpina e Toggenburg; $M_{k}$, efeito fixo do $k_{\text {ésimo }}$ mês do parto; $\mathrm{k}$, janeiro a dezembro; $\mathrm{A}_{1}$, efeito fixo do $\mathrm{l}_{\text {ésimo }}$ ano do parto; $1=1995,1996,1997 ; \mathrm{T}_{\mathrm{m}}$, efeito fixo do $\mathrm{m}_{\text {ésimo }}$ tipo de parto; $\mathrm{m}$, simples e múltiplo; $\mathrm{S}_{\mathrm{n}}=$ efeito fixo do $\mathrm{n}_{\text {ésimo }}$ sexo da cria; $\mathrm{n}$, macho e fêmea; $b_{l}$, coeficiente linear de regressão de peso da cabra à cobertura; $b_{2}$,coeficiente quadrática de regressão de peso da cabra à cobertura; $\mathrm{P}_{\mathrm{ijklmn}}$, peso da cabra à cobertura;
$\overline{\mathrm{P}}$, peso médio à cobertura; $\mathrm{e}_{\mathrm{ijklmn}}$, erro aleatório, normal, independentemente distribuído com médio zero e variância $\sigma^{2}$, associado a cada observação.

As herdabilidades das características por raça foram estimadas usando o programa MTDFREML, com o seguinte modelo animal. No caso da raça Parda Alpina foram utilizados os mesmos reprodutores nos dois capris.

$$
\mathrm{y}=\mathrm{Xb}+\mathrm{Z}_{1} a+e
$$

em que y é vetor de observações da idade ao primeiro parto, do intervalo de partos, da produção de leite e da duração da lactação; $X$, matriz de incidência dos efeitos fixos do vetor $b ; Z_{1}$, matrizes de incidência dos efeitos aleatórios do vetor a; $a$, vetor de efeitos aleatórios genéticos aditivos; $e$, vetor de erros aleatórios associados a cada observação.

\section{Resultados e Discussão}

Na Tabela 1 são apresentados os resultados das análises, demonstrando que a idade ao primeiro parto foi influenciada $(\mathrm{P}<0,01)$ pela raça e pelo tipo de parto. Capril, raça, mês do parto e sexo da cria exerceram influências significativas $(\mathrm{P}<0,05)$ sobre o intervalo de parto. Somente a raça influenciou significativamente $(\mathrm{P}<0,05)$ a produção de leite total. Entretanto, nenhum fator estudado influenciou o período de gestação e a duração da lactação.

Foram observadas grandes diferenças entre raças quanto à idade ao primeiro parto com médias de $22,52 \pm 10,53$ para raça Saanen, 34,22 $\pm 23,92$ para Parda Alpina, 45,10 \pm 30,97 para Toggenburg e $39,35 \pm 16,16$ meses para os mestiços, assim como para o intervalo de parto com médias de 394,53 \pm 112,16 para raça Saanen, 299,40 \pm 40,02 dias para Parda Alpina, 445,00 \pm 1,41 para Toggenburg e $207,00 \pm 39,59$ dias para os mestiços (Tabela 2), sendo que a raça Saanen se destacou por apresentar menor idade ao primeiro parto e os mestiços menor intervalo de parto.

GALINA et al. (1995), trabalhando com cabras leiteiras mexicanas, obtiveram média de 14 meses de idade ao primeiro parto, mostrando que as cabritas foram acasaladas com 9 meses de idade. Na região Oeste da África, WILSON e LIGHT (1986) registraram 16,18 meses, como valores médios para IPP. Já KENNEDY et al. (1982), em cabras Parda Alpina, Saanen e Toggenburg encontraram valores de 17,1 meses para a IPP. As médias apresentadas acima revelam que, quando se consegue o primeiro parto 
Tabela 1 - Resumo da análise de variância para idade ao primeiro parto (IPP), intervalo de partos (IP), período de gestação (PG), produção de leite total (PLT) e duração da lactação (DL) em cabras das raças Saanen, Parda Alpina, Toggenburg e mestiças nos dois criatórios

Table 1 - Summary of analysis of variance for age at first kidding (AFK), kidding interval (KI), gestation period (GP), total milk production (TMP) and lactation length (LL) in goats of the Saanen, Parda Alpine and Toggenburg breeds and their crossbreds

\begin{tabular}{|c|c|c|c|c|c|}
\hline Fonte de variação & IPP & IP & PG & PLT & DL \\
\hline Source of variation & $A F K$ & $K I$ & $G P$ & $T M P$ & $L L$ \\
\hline $\begin{array}{l}\text { Capril } \\
\text { Farm }\end{array}$ & 774717,1 & $8794,3^{*}$ & 30,4 & 52243,2 & 4451,5 \\
\hline $\begin{array}{l}\text { Raça } \\
\text { Breed }\end{array}$ & $1823480,7^{* *}$ & $21324,2 *$ & 37,2 & $160537,1^{*}$ & 3064,5 \\
\hline $\begin{array}{l}\text { Mês do parto } \\
\text { Month of kidding }\end{array}$ & 304902,6 & $13945,1^{*}$ & 38,4 & 39239,2 & 3816,7 \\
\hline $\begin{array}{l}\text { Ano do parto } \\
\text { Year of kidding }\end{array}$ & 638720,8 & 3702,1 & 7,6 & 41516,4 & 4606,6 \\
\hline $\begin{array}{l}\text { Tipo de parto } \\
\text { Type of parturation }\end{array}$ & $1892173,0^{* *}$ & 1238,6 & 78,9 & 33148,3 & 5896,9 \\
\hline $\begin{array}{l}\text { Sexo da cria } \\
\text { Sex of the kid }\end{array}$ & 124445,6 & $7932,3^{*}$ & 16,9 & 6316,2 & 5,97 \\
\hline $\begin{array}{l}\text { Peso de cobertura } \\
\text { Weight at matting }\end{array}$ & 278849,4 & 393,1 & 48,5 & 260,9 & 1061,8 \\
\hline $\begin{array}{l}\mathrm{R}^{2} \\
\mathrm{CV}(\%)\end{array}$ & $\begin{array}{r}0,35 \\
64,09\end{array}$ & $\begin{array}{r}0,97 \\
10,77\end{array}$ & $\begin{array}{l}0,30 \\
3,92\end{array}$ & $\begin{array}{r}0,44 \\
43,01\end{array}$ & $\begin{array}{r}0,42 \\
28,40\end{array}$ \\
\hline
\end{tabular}

entre 14 e 17 meses, esses animais são economicamente mais viáveis em relação às cabras que têm o primeiro parto a partir dos 18 meses. Isto é importante do ponto de vista reprodutivo, pois os animais mostram sua eficiência em desenvolvimento, conseqüência de bom manejo na fase de crescimento, gerando coberturas e partos precoces com aproveitamento pleno do animal na sua vida útil.

O alto valor médio da idade ao primeiro parto com $32,91 \pm 18,52$ meses (Tabela 2 ) talvez tenha sido influenciado pelo manejo da primeira cobertura, já que não faz sentido uma cabra conceber pela primeira vez por volta dos 23 meses de idade. GONÇALVES
(1996) relatou que, para conseguir resultados econômicos razoáveis, as cabritas devem alcançar o primeiro parto com 12 meses, independente do fotoperiodismo. As diferenças encontradas neste estudo, provavelmente, decorrem do manejo, principalmente o reprodutivo, talvez influenciado pela primeira cobertura das fêmeas, levando a reflexos diretos nas variações de idades ao parto. As variações do fotoperiodismo também podem ter influenciado as taxas de concepção e, conseqüentemente, as idades ao primeiro parto. Como os animais utilizados neste estudo são poliéstricos estacionais, se não estiverem submetidos a um manejo adequado, a eficiência reprodutiva será afetada.

Tabela 2 - Média de quadrados mínimos da idade ao primeiro parto (meses), intervalo de partos (dias) e período de gestação (dias) em cabras das raças Saanen, Parda Alpina, Toggenburg e mestiças

Table 2 - Least square means of age at first kidding (months), kidding interval (days) and gestation period (days) in goats of the Saanen, Alpine and Toggenburg breeds and their crossbreds

\begin{tabular}{lccc}
\hline & $\begin{array}{c}\text { Média } \pm \mathrm{dp} \\
\text { Average } \pm \text { sd }\end{array}$ & $\begin{array}{c}\text { Média } \pm \mathrm{dp} \\
\text { Average } \pm \text { sd }\end{array}$ & $\begin{array}{c}\text { Média } \pm \mathrm{dp} \\
\text { Average } \pm \text { sd }\end{array}$ \\
Raça & $\begin{array}{c}\text { Idade ao primeiro parto (meses) } \\
\text { Age at first kidding(months) }\end{array}$ & $\begin{array}{c}\text { Intervalo de partos (dias) } \\
\text { Kidding interval (days) }\end{array}$ & $\begin{array}{c}\text { Período de gestação (dias) } \\
\text { Gestation period (days) }\end{array}$ \\
\hline Saanen & $22,52 \pm 10,53$ & $394,53 \pm 112,16$ & $151,32 \pm 3,62$ \\
Parda Alpina & $34,22 \pm 23,92$ & $299,40 \pm 40,02$ & $153,28 \pm 7,81$ \\
Toggenburg & $45,10 \pm 30,97$ & $445,00 \pm 1,41$ & $150,00 \pm 2,49$ \\
Mestiços & $39,35 \pm 16,16$ & $207,00 \pm 39,59$ & $149,91 \pm 8,57$ \\
Crossbred & & & \\
Médiageral & $32,91 \pm 18,52$ & $360,45 \pm 38,82$ & $151,47 \pm 5,94$ \\
Overall mean & & &
\end{tabular}

$\mathrm{dp}=$ desvio-padrão.

$s d=$ standard deviation 
A média obtida para os intervalos de partos foi de $360,45 \pm 38,82$ dias, para todos os animais estudados (Tabela 2). Valores aproximados foram citados por GALINA et al. (1995), na região semi-árida do México, onde estudaram a performance reprodutiva de cabras mexicanas; esse estudo revelou valores para IP de 347 dias, enquanto, em Alagoas, CÂNCIO et al. (1992), estudando animais da raça Saanen e mestiços de Saanen com Marota, não encontraram diferenças significativas entre os grupos estudados e relataram média de 367 dias. Já GONÇALVES et al. (1996), na região Sudeste do Brasil, trabalhando com animais das raças Parda Alpina, Saanen e Toggenburg, obtiveram IP de 339,9 dias, média inferior à encontrada com os animais deste estudo. Por outro lado, NDLOVU e SIMELA (1996) encontraram médias inferiores em cabras Africanas, com valor médio de 315 dias.

Verificou-se que, ao longo dos anos estudados, não houve decréscimo na média dos intervalos de partos. Isto pode ser atribuído ao manejo, uma vez que o criador prefere prolongar a cobertura da cabra, mantendo-a em lactação, devido ao alto preço do leite no mercado local. Também, as observações de cio ocorrem uma única vez ao dia, elevando as falhas de identificação e possíveis coberturas no momento certo. Portanto, os valores das médias dos intervalos obtidos neste estudo foram um pouco inferiores a 365 dias, revelando razoável desempenho reprodutivo, levando em consideração que os animais estudados apresentam grande sensibilidade às variações de fotoperíodo.

O efeito da raça sobre o intervalo de partos não foi observado em nenhum trabalho na literatura revisada. O mesmo não ocorre com a influência do mês do parto, pois existem vários trabalhos que citam diferenças, como TERRIL e FOOTE (1987), CÂNCIO et al. (1992), GALINA et al. (1995) e GONÇALVES (1996). A influência de raça sobre os intervalos de partos verificada (Tabela 1) talvez seja reflexo da constituição genética, já que os animais foram criados sob as mesmas condições.

Houve efeito significativo do mês do parto $(\mathrm{P}<0,05)$ sobre o intervalo de partos, mostrando alguma variação na duração dos intervalos (Tabela 1). O fato de o mês do parto ter influência sobre o intervalo de partos pode ser explicado pelas variações do fotoperíodo, pelo manejo e pela alimentação.

A análise de variância para período de gestação não revelou efeito significativo em nenhuma variável (Tabela 1). De maneira geral, os resultados diferem dos relatados por AMOACH et al. (1996) e
MEDEIROS et al. (1997), os quais encontraram influências de raça, tipo de parto, mês e ano do parto e ordem do parto.

CÂNCIO (1991), estudando alguns aspectos reprodutivos de animais das raças Saanen e mestiças Saanen com Marota, relatou que o período de gestação médio foi de 146 dias. A média obtida neste estudo para período de gestação foi de 151,47 $\pm 5,94$ dias nos dois capris (Tabela 2), para todas as raças estudadas, estando os valores superiores aos encontrados por CÂNCIO (1991).

A influência da raça sobre a produção de leite, observada neste estudo (Tabela 1), está de acordo com os relatos feitos por ALDERSON E POLLAK (1980), ILOEJE et al. (1981) e BARBIERI et al. (1990b). A superioridade da produção total de leite da raça Saanen sobre a Parda Alpina, Toggenburg e mestiços também foi encontrada por ALDERSON e POLLAK (1980) nos Estados Unidos.

Na Tabela 1, observa-se que o mês e ano do parto, o tipo do parto e o sexo da cria não apresentaram diferença significativa em relação à produção de leite nos animais estudados.

A raça Saanen apresentou produção de leite total média superior às demais raças com valores de $563,64 \pm 256,41 \mathrm{~kg}$ contra 513,88 $\pm 193,53,338,32 \pm$ 94,41 e $319,97 \pm 104,80 \mathrm{~kg}$ para as raças Parda Alpina, Toggenburg e mestiços, respectivamente (Tabela 3).

A média para produção diária de leite foi de $2,34 \pm 1,17 \mathrm{~kg} /$ dia para os dois capris (Tabela 3 ). Levando em consideração o tipo de manejo, os resultados foram satisfatórios. Valores próximos deste estudo foram encontrados por BARBIERI et al. (1990b) na região Nordeste do Brasil, com médias de 2,3 e 1,6 kg/dia para Saanen e Parda Alpina, respectivamente. Entretanto, melhores resultados foram relatados por KENNEDY et al. (1982) nos Estados Unidos, com valores médios de 2,6 kg/dia para Saanen, Parda Alpina e Toggenburg e os encontrados por GONÇALVES (1996), na região Sudeste do Brasil, com médias de 2,90 kg/dia para raça Saanen, $2,19 \mathrm{~kg} /$ dia para Parda Alpina e 1,80 kg/dia para raça Toggenburg.

Foi observado neste estudo que a produção média de leite de todo o rebanho se apresenta dentro dos padrões encontrados para estas raças no Brasil. De acordo com GONÇALVES (1996), as raças exóticas criadas no Brasil não apresentam melhores resultados produtivos, em virtude da falta de um programa de seleção e melhoramento destes animais. 
Tabela 3 - Médias de quadrados mínimos da produção de leite total $(\mathrm{Kg})$, duração da lactação (dias) e produção de leite por dia $(\mathrm{kg})$ em cabras das raças Saanen, Parda Alpina, Toggenburg e mestiças

Table 3 - Least square means for total milk production ( $\mathrm{kg}$ ), lactation length (days) and daily milk production ( $\mathrm{kg}$ ) in goats of the Saanen, Alpine and Toggenburg breeds and their crossbreds

\begin{tabular}{lccc}
\hline & $\begin{array}{c}\text { Média } \pm \mathrm{dp} \\
\text { Average } \pm s d\end{array}$ & $\begin{array}{c}\text { Média } \pm \mathrm{dp} \\
\text { Average } \pm s d\end{array}$ & $\begin{array}{c}\text { Média } \pm \mathrm{dp} \\
\text { Average } \pm \text { sd }\end{array}$ \\
Raça & $\begin{array}{c}\text { Produção de leite total }(\mathrm{kg}) \\
\text { Total milk production }(\mathrm{kg})\end{array}$ & $\begin{array}{c}\text { Produção de leite /dia (kg/dia) } \\
\text { Daily milk production (kg/day) }\end{array}$ & $\begin{array}{c}\text { Duração da lactação (dias) } \\
\text { Lactation length }(\text { days) }\end{array}$ \\
\hline Saanen & $563,64 \pm 256,41$ & $2,34 \pm 0,63$ & $238,43 \pm 72,88$ \\
Parda Alpina & $513,88 \pm 193,53$ & $2,01 \pm 0,61$ & $238,88 \pm 71,68$ \\
Toggenburg & $338,32 \pm 94,41$ & $1,70 \pm 0,52$ & $211,88 \pm 54,97$ \\
Mestiços & $319,97 \pm 104,80$ & $1,79 \pm 0,53$ & $190,14 \pm 50,32$ \\
Crossbred & & & $246,95 \pm 69,00$ \\
Média geral & $505,29 \pm 226,08$ & $2,34 \pm 1,17$ & \\
Overall mean & & & \\
\hline
\end{tabular}

$\mathrm{dp}=$ desvio-padrão.

sd $=$ standard deviation

As médias de duração da lactação para todos os animais foram $246,95 \pm 69,00$ dias nos dois capris (Tabela 3). As médias por raça são apresentadas na Tabela 3. Observou-se que os valores médios da duração da lactação foram inferiores aos 305 dias, revelando bom desempenho reprodutivo e, conseqüentemente, produtivo, uma vez que os animais estudados são sensíveis às variações do fotoperíodo.

Resultados semelhantes foram relatados por KENNEDY et al. (1982) e GONÇALVES (1996), que, trabalhando com as mesmas raças desde estudo, encontraram valores de 212, 266 e 236 dias de lactação, respectivamente.

Capris, raça, ano e mês do parto, tipo de parto, sexo da cria e peso de cobertura neste estudo não afetaram significativamente a duração da lactação (Tabela 1). Para o tipo de parto, os resultados foram semelhantes aos relatados por BARBIERI et al. (1990a). BARBIERI et al. (1990b) e GONÇALVES (1996) constataram influência significativa da estação de parto sobre a duração da lactação. O sexo da cria influenciando o período de lactação não foi um efeito encontrado na literatura consultada.

As médias ajustadas das características para os efeitos do modelo analisado são apresentadas na Tabela 4. Observa-se grande variação entre todos os fatores considerados, possivelmente em decorrência de deficiência no manejo e controle dentro dos capris. É importante salientar que isto ocorre, na maioria dos casos, devido à falta de planejamento nas propriedades, uma vez que os animais são criados em confinamento sendo elevado a possibilidade de controlar determinados efeitos.
As estimativas de herdabilidade $\left(\mathrm{h}^{2}\right)$ para a idade ao primeiro parto por raça foram 0,10 para Saanen, 0,08 para Parda Alpina e 0,04 para Toggenburg, valores considerados como de baixa magnitude. Estes valores foram inferiores aos obtidos por GONÇALVES (1996), com média de 0,22 para animais das raças Saanen, Parda Alpina e Toggenburg, e aos relatados por RIBEIRO et al. (1997b), com valor de 0,14 para animais Saanen. Como os capris estudados adotam critérios de peso à cobertura entre $32 \mathrm{a} 35 \mathrm{~kg}$, pode ocorrer pequeno atraso para o animal chegar a este peso, implicando em acréscimo na idade ao primeiro parto. Portanto, a herdabilidade desta característica sofre com a influência da taxa de crescimento do animal, podendo causar viés na estimativa de herdibilidade.

Para o intervalo de partos, as estimativas de herdabilidade $\left(\mathrm{h}^{2}\right)$ foram 0,16 para Saanen, 0,02 para Parda Alpina e 0,05 para Toggenburg. Estes valores também são baixos e inferiores aos relatados por SINGH et al. (1970) e GONÇALVES (1996). Foram, no entanto, superiores aos resultados relatados por RIBEIRO et al., (1997b) para a raça Saanen. Já para produção de leite os valores de $\mathrm{h}^{2}$, foram 0,06 para Saanen, 0,11 para Parda Alpina e 0,12 para Toggenburg. Estas estimativas estão próximas ao valor de 0,09 para raça Saanen encontrado por RIBEIRO et al. (1997a) e inferiores às encontradas por ILOEJE et al. (1981), KENNEDY et al. (1982) e GONÇALVES (1996) em caprinos das raças Parda Alpina, Saanen e Toggenburg.

As estimativas de herdabilidades para a duração da lactação foram 0,07 para Saanen, 0,07 para Parda 
Rev. bras. zootec.

Tabela 4 -Média de quadrados mínimos da idade ao primeiro parto (IPP), intervalo de partos (IP), duração da lactação (DL) e produção de leite total (PLT), conforme as variáveis analisadas

Table 4 - Least square means for age at first kidding (AFK), kidding interval (KI), lactation length (LL) and total milk production (TMP), according to the studied variable

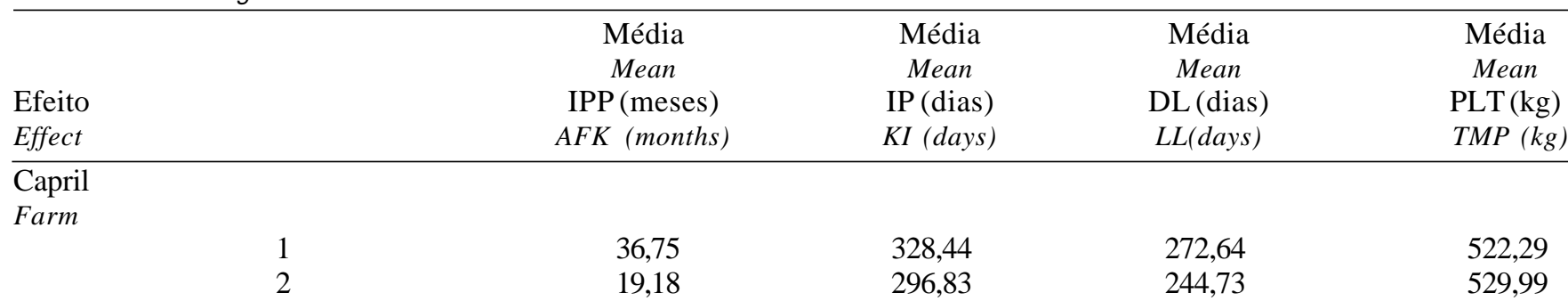

Mês do parto

Month of kidding

Ano do parto

Janeiro
January
Fevereiro
February
Março
March
Abril
April
Maio
May
Junho
June
Julho
July
Agosto
August
Setembro
September
Outubro
October
Novembro
November
Dezembro
December

30,27

255,61

268,55

528,76

31,97

190,83

282,64

576,08

23,32

395,56

217,57

372,96

30,70

292,48

232,59

479,71

29,21

277,45

264,49

669,20

18,53

298,20

280,78

525,61

24,95

325,67

249,65

538,73

25,77

362,46

273,22

536,66

27,79

297,54

268,32

532,12

28,76

325,23

264,71

503,78

28,27

300,79

259,94

493,56

36,00

429,70

246,77

556,49

Year of kidding

$\begin{array}{ll}1995 & 26,51 \\ 1996 & 29,10 \\ 1997 & 28,26\end{array}$

289,85

335,42

266,57

527,45

29,10

250,80

524,54

Tipo de parto

Type of parturition

\begin{tabular}{lllll}
$\begin{array}{l}\text { Simples } \\
\text { Single }\end{array}$ Duplo & 26,29 & 321,56 & 254,41 & 498,89 \\
$\begin{array}{l}\text { Twin } \\
\text { Triplo } \\
\text { Triple }\end{array}$ & 31,24 & 329,43 & 256,16 & 519,10 \\
& 26,37 & 286,91 & 265,48 & 560,44 \\
\hline
\end{tabular}

Alpina e 0,03 para Toggenburg. RIBEIRO et al. (1997a) encontraram estimativas próximas a estas na raça Saanen, com valor de 0,06.

Observou-se que as estimativas das $\mathrm{h}^{2}$ encontradas neste estudo podem estar subestimadas, pois o número de informações disponíveis, além de limitado, foi coletado em apenas dois rebanhos no período de três anos.

\section{Conclusões}

A escolha da raça é muito importante em determinar o sucesso do empreendimento. Neste estudo, a raça Saanen mostrou maior produção de leite e as menores idades ao primeiro parto.

Os fatores ambientais foram importantes nas 
características estudadas e devem ser utilizados como fatores de ajustes. Os fatores capril e mês do parto foram os mais prevalecentes, evidenciando talvez mudanças no manejo ao longo dos anos.

As estimativas de herdabilidade indicaram pouca variabilidade genética nas características estudadas, o que talvez possa ser explicado pelo reduzido número de dados nos dois rebanhos ou por viés causados pelo manejo dos animais.

Os níveis de produção deste estudo apresentam-se dentro das médias encontradas na literatura brasileira, mas mostram que existe oportunidade para a melhoria nos índices zootécnicos, particularmente a idade primeiro parto e o intervalo de partos.

\section{Referências Bibliográficas}

ALDERSON, A., POLLAK, J. 1980. Age season adjustment factors for milk and fat of dairy goats. J. Dairy Sci., 63:148-151.

AMOACH, E.A., GELAYE, S., GUTHRIE, P. et al. 1996. Breeding season and aspects of reproduction of female goats. J. Anim. Sci., 74:723-728.

BARBIERI, M.E., FIGUEIREDO, E.A.P., SIMPLÍCIO, A.A. Produção de leite em cabras meio sangue Parda Alpina e Moxotó, em Sobral, Ceará. In: REUNIÃO ANUAL DA SOCIEDADE BRASILEIRA DE ZOOTECNIA, 27, 1990, Campinas. Anais... Campinas, 1990a. p.408.

BARBIERI, M.E., VASCONCELOS, A.S.E., SIMPLÍCIO, A.A., et al. Avaliação produtiva de cabras leiteiras das raças Saanen, Parda Alpina e Anglo-Nubiana. In: REUNIÃO ANUAL DA SOCIEDADE BRASILEIRA DE ZOOTECNIA, 27, 1990. Campinas. Anais... Campinas, 1990b. p.410.

BOLDMAN, K.G., KRIESE, L.A., VAN VLECK, D.L. et al. 1993. A manual for use of MTDFREML a set of programs to obtain estimates of variances and covariances. ARS, USDA. 120p.

CÂNCIO, C.R.B. Eficiência reprodutiva de cabras, Saanen, Marota e mestiças (F1) Saanen-Marota no semi-árido alagoano. Recife, PE: UFPE, 1991, 57p. Dissertação (Mestrado) - Universidade Federal Rural de Pernambuco, 1991.

CÂNCIO, C.R.B., CASTRO, R.S., COELHO, L.A. et al. 1992. Idade ao primeiro parto, intervalo entre partos e produção leiteria de cabras Saanen, Marota e mestiças em Alagoas. Pesq. Agropec. Bras., 27(1):53-59.

CHEMINEAU, P., BERTHELOT, X., MALPAUX, B. et al. 1993. La maitrise de la reproduction par la photopériode et la mélatonine chez les mammífères d'élevage. Casch. Agric., 2:81-92.

GALINA, M.A., SILVA, E., MORALES, R. et al. 1995. Reproductive performance of Mexican dairy goats under various management systems. Small Rumin. Res., 18:249-253.

GONÇALVES, H.C., SILVA, M.A., MARTINS, E.N. et al. Fatores genéticos e de meio na idade ao primeiro parto de caprinos no Brasil. In: REUNIÃO ANUAL DA SOCIEDADE BRASILEIRA DE ZOOTECNIA, 33, 1996. Fortaleza. Anais... Fortaleza: SBZ, 1996. p.163-165.

GONÇALVES, H.C. Melhoramento genético de caprinos leiteiros. In: ENCONTRO NACIONAL PARA O DESENVOLVIMENTO DA ESPÉCIE CAPRINA, 4, 1996, Pirassununga, Anais... Pirassununga, 1996. p.21-68.

ILOEJE, M.U., VAN VLECK, L.D. 1978. Genetics of dairy goats. A Review. J. Dairy Sci., 61:1521-1528.

ILOEJE, M.U., VAN VLECK, L.D., WIGGANS, G.R. 1981. Components of variance for milk and fat yields in dairy goats. J. Dairy Sci., 64:2290-2293.

KENNEDY, B.W., FINLEY, C.M., BRADFORD, G.E. 1982. Phenotypic and genetic relationships between reproduction and milk prodution in dairy goats. J. Dairy Sci., 65(12):2373-2383.

MEDEIROS, R.G., PIMENTA FILHO, E.C., LEITE, S.V.F. et al. Fatores que afetam o período de gestação de cabras nativas e exóticas no semi-árido. In: REUNIÃO ANUAL DA SOCIEDADE BRASILEIRA DE ZOOTECNIA, 34, 1997, Juiz de Fora, Anais... Juiz de Fora, 1997. p.278-280.

NDLOVU, L.R., SIMELA, L. 1996. Effect of season of birth and sex of kid on the production of live weaned single born kids in smallholder East African goat flocks in North East Zimbabwe. Small Rumin. Res., 22:1-6.

RIBEIRO, A. C., LUI, J. F., QUEIROZ, S. A. et al. Estimativas dos parâmetros genéticos e fenótípicos de características produtivas em caprinos da raça Saanen. In: REUNIÃO ANUAL DA SOCIEDADE BRASILEIRA DE ZOOTECNIA, 34, 1997, Juiz de Fora. Anais... Juiz de Fora, 1997a. v.3, p. 275-277.

RIBEIRO, A.C., LUI, J.F., QUEIROZ, S.A. et al. Estimativas dos parâmetros genéticos e fenótípicos de características reprodutivas em caprinos da raça Saanen. In: REUNIÃO ANUAL DA SOCIEDADE BRASILEIRA DE ZOOTECNIA, 34, 1997, Juiz de Fora. Anais... Juiz de Fora, 1997b. v.3, p.281-283.

RUVUNA, F., CARTWRIGHT, T.C., OKEYO, M.A. et al. Characterization of indigenuos goats of Kenya for dual purpose potencial 2. Weights and pospartum potencial interval. In. INTERNATIONAL CONFERENCE ON GOATS, 4. Brasília, 1987. Proceedings... Brasília, 1987, 2:1314.

SAS User's guide: Statistics. SAS Institute Inc., Cary, North Caroline. 1985.

SIMPLÍCIO, A.A., MACHADO, R., ALVES, J.A. 1990. Manejo reprodutivo de caprinos em regiões tropicais. In: Caprinocultura e Ovinocultura, Sociedade Brasileira de Zootecnia, Piracicaba: FEALQ. p.34-56.

SIMPLÍCIO, A.A., RIERA, G.S., NUNES, J.F. et al. 1986. Frequence and durantion of estrous cycle and period in genetically non-descript (SRD) type of goats in the tropical northeast of Brazil. Pesq. Agropec. Bras., 21(5):535-540.

SINGH, R.N., ACHARYA, R.M., BISWAS, D.K. 1970. Evaluation of genetic and non-genetic factors affecting some economic traits in goats. Acta Agric. Scand., 20:10-14.

TERRIL, C.E., FOOTE, W.C. Estimating reproductive performance in goat. In: INTERNATIONAL CONFERENCE ON GOATS, 4, Brasília, 1987. Proceedings... Brasília, 1987. v. 1, p. $577-583$.

WILSON, R.T., LIGHT, D. 1986. Livestock production in central Mali: economic characters and productivity indices for traditionally managed goats end sheep. J. Anim. Sci., 62:567-575.

WOOD, P.D.P. 1980. Breed variation in the shape of the lactation curve of cattle and their implications for efficiency. Anim. Prod., 34:133-141.

Recebido em: 15/05/00

Aceito em: 29/09/00 